\title{
Glueballs at finite temperature from AdS/QCD
}

\author{
Alex S. Miranda ${ }^{a b *}$, C. A. Ballon Bayona ${ }^{c \dagger}$, Henrique Boschi-Filho ${ }^{\text {bł }}$ and Nelson R. F. Braga ${ }^{\mathrm{b} \S}$ \\ ${ }^{a}$ Laboratório de Astrofísica Teórica e Observacional \\ Departamento de Ciências Exatas e Tecnológicas \\ Universidade Estadual de Santa Cruz, 45650-000, Ilhéus, BA, Brazil \\ b Instituto de Física, Universidade Federal do Rio de Janeiro, \\ Caixa Postal 68528, RJ 21941-972, Brazil \\ ${ }^{\mathrm{c}}$ Centro Brasileiro de Pesquisas Físicas, Rua Dr. Xavier Sigaud 150, \\ Urca, 22290-180, Rio de Janeiro, RJ, Brazil
}

Inspired in the AdS/CFT correspondence, a variety of holographic phenomenological models have been proposed in the last years to describe non-perturbative aspects of strong interactions. These models are denominated as AdS/QCD. In this work we review the use of the AdS/QCD soft-wall model to investigate the spectrum of scalar glueballs at finite temperature. The scalar glueball states are identified as the poles of the retarded correlation function of the glueball operator. In the gauge/gravity duality, these poles are determined by the quasinormal spectrum of a massless scalar field propagating in the bulk geometry that consists on an $\mathrm{AdS}_{5}$ black hole with a background dilaton field. We discuss some results for masses and decay widths of scalar glueballs in the plasma phase and analyse how these quantities evolve with temperature and momentum.

\section{Introduction}

The strong interactions are described by QCD which is a $\mathrm{SU}(3)$ gauge theory. At high energies, the coupling constant is small and the theory can be treated with perturbative methods. When one considers processes involving high energies one finds very good agreement between theoretical predictions and experimental results. However, many important aspects of strong interactions, like mass generation and quark confinement, are not yet completely understood because they involve the low-energy non-perturbative regime of QCD. So, it is important to look for alternative tools to deal with this non-perturbative regime. A traditional approach is to use lattice simulations that still do not furnish a complete description of low-energy QCD. A more recent proposal is to construct phenomenological models for

\footnotetext{
*email: astmiranda@if.ufrj.br

†email: ballon@cbpf.br

¥email: boschi@if.ufrj.br

§email: braga@if.ufrj.br
}

strong interactions based on the AdS/CFT correspondence [123]. These models are presently known as AdS/QCD. The simplest model, known as hard wall, consists in using an AdS slice with a size related to an infrared cut-off in the gauge theory. This model was used to estimate hadron masses 456789]. It presents confinement at zero temperature [10] and deconfinement at high temperatures [1]. Another interesting AdS/QCD model that leads to linear Regge trajectories is known as soft wall. In this model a non-uniform background dilaton field plays the role of an effective infrared cut-off. This model was also used to calculate hadronic masses 121314 . The confinement/deconfinement thermal phase trasition in both models was studied in Refs. 151617. It was found that there is a Hawking-Page gravitational transition such that the space with a black hole is thermally favored at high temperatures, while below some critical (model-dependent) value, the thermally favored space is a pure AdS.

In this paper we are going to review some recent 
progress in using the soft-wall AdS/QCD model to compute the spectrum of scalar glueballs in the finite-temperature plasma phase of QCD 18 (See also Ref. 19]). We consider the AdS blackhole spacetime for all temperature values. As commented above, for temperatures lower than a critical value the black hole is thermodynamically unstable and can be interpreted as a supercooled Yang-Mills plasma.

The massless scalar field in the 5 -d bulk is dual to scalar glueballs in the 4-d boundary theory and the black-hole quasinormal modes correspond to the poles of the retarded correlation function of the operator $\mathcal{O}=\operatorname{Tr}\left(F^{2}\right)$ that creates scalar glueball states. With a numerical analysis, we obtain the real and imaginary parts of the quasinormal mode frequencies as a function of the temperature and the spatial momentum. The numerical computations at high temperatures were performed using series solutions of the equation of motion. At low temperatures, we found problems with the convergence of the series so it was necessary to use an alternative approach. We have chosen the Breit-Wigner method which has been recently applied to compute black-hole quasinormal modes in Ref. 20. We calculate the retarded Green's function of scalar glueballs and verify that their poles coincide with the quasinormal mode frequencies. The imaginary part of the retarded Green's function gives the spectral function, which we also analyze in this article.

Other recent results in scalar glueballs can be found in 21]. For a general review on glueballs see 22$]$.

\section{The soft-wall model at finite tempera- ture}

Our main objective in this paper is to review recent work [18] on the spectrum of scalar glueballs at finite temperature in the soft-wall AdS/QCD model. For this purpose, it is considered a dilaton field $\Phi=c z^{2}$ in a finite-temperature background that consists of an AdS black-hole spacetime with metric

$d s^{2}=e^{2 A}\left[-f d t^{2}+d x^{i} d x^{i}+f^{-1} d z^{2}\right]$, where $f=1-\left(z / z_{h}\right)^{4}, A=-\ln (z / L)$ and $L$ denotes the AdS curvature radius. The coordinate $z$ is defined in the range $0 \leq z \leq z_{h}$ and $z=0$ corresponds to the boundary of the spacetime. The parameter $z_{h}$ indicates the position of the event horizon which is related to the black-hole Hawking temperature $T$ by the relation $z_{h}=1 / \pi T$, where $T$ also represents the temperature of the boundary field theory.

The $\mathrm{AdS}_{5}$ black-hole spacetime considered here is associated to three distinct phases, each one corresponding to a range of values of the temperature parameter $\widetilde{T}=\pi T / \sqrt{c}$. The thermal competition between the black hole and thermal AdS space is such that there is a Hawking-Page transition between spaces at $\widetilde{T}_{c}^{2} \approx 2.38644$. This transition is interpreted in the boundary field theory as a confinement/deconfinement phase transition. The black hole spacetime is the dominant configuration for $\widetilde{T}>\widetilde{T}_{c}$, while the thermal AdS space is the dominant one for low temperature values. Hence, for $\widetilde{T}<\widetilde{T}_{c}$, the plasma will be in a metastable (unstable) phase corresponding to a positive (negative) sign of the black-hole specific heat $\mathcal{C}_{B H}$. It was shown in Ref. 18, that $\mathcal{C}_{B H}>0$ for $\widetilde{T}^{2} \gtrsim 0.75$ and $\mathcal{C}_{B H}<0$ for $\widetilde{T}^{2} \lesssim 0.75$.

In the soft-wall model [12, the dilaton background field $\Phi(z)=c z^{2}$ interacts with the bulk fields through the replacement of the action integrals: $\int d^{5} x \sqrt{-g} \mathcal{L} \Rightarrow \int d^{5} x \sqrt{-g} e^{-\Phi} \mathcal{L}$. In particular, the action for the massless scalar field $\phi$ is given by:

$S=-\frac{\pi^{3} L^{5}}{4 \kappa_{10}^{2}} \int d^{5} x \sqrt{-g} e^{-\Phi} g^{M N} \partial_{M} \phi \partial_{N} \phi$,

where $g_{M N}$ is the black-hole metric given by (1) and $\kappa_{10}$ is the ten-dimensional gravitational constant. The indices $M, N$ run over $0,1, \ldots, 4$ where $x^{\mu}(\mu=0, \ldots, 3)$ are the $4 d$ boundary coordinates and $x^{4}=z$ is the extra radial coordinate.

\section{The Schrödinger equation}

We do not consider here the backreaction of the dilaton $\Phi$ and of the scalar field $\phi$ on the metric. Then, from the action (2), we obtain a linearized 
equation of motion for $\phi$ :

$\frac{e^{\Phi}}{\sqrt{-g}} \partial_{z}\left(\sqrt{-g} e^{-\Phi} g^{z z} \partial_{z} \phi\right)+g^{\mu \nu} \partial_{\mu} \partial_{\nu} \phi=0$.

After Fourier decomposition of $\phi$ with respect to the coordinates $x^{\mu}$, Eq. (3) becomes

$e^{B} f \partial_{z}\left(e^{-B} f \partial_{z} \phi\right)+\left(\omega^{2}-f q^{2}\right) \phi=0$,

where $k_{\mu}=\left(-\omega, q_{i}\right), \quad q^{2}=\sum_{i=1}^{3} q_{i}^{2}$ and $B=\Phi-3 A=c z^{2}+3 \ln (z / L)$.

The foregoing equation takes the form of a onedimensional Schrödinger equation when written in terms of the new variable $\psi=e^{-B / 2} \phi$ :

$\partial_{r_{*}}^{2} \psi+\omega^{2} \psi=V \psi$

where $r_{*}$ is the tortoise radial coordinate, defined in such a way that $d z / d r_{*}=-f$, and the effective potential $V$ is given by

$$
\begin{aligned}
V= & \frac{f}{z^{2}}\left[q^{2} z^{2}+\frac{15}{4}+\frac{9}{4} \frac{z^{4}}{z_{h}^{4}}\right. \\
& \left.+2 c z^{2}\left(1+\frac{z^{4}}{z_{h}^{4}}\right)+c^{2} z^{4} f\right] .
\end{aligned}
$$

The general solution of equation (5) can be written as a linear combination of a normalizable solution $\psi_{1}$ and a non-normalizable solution $\psi_{2}$. These wave functions have the following asymptotic form near the boundary $z=0$ :

$$
\begin{aligned}
\psi_{1}= & z^{5 / 2}\left[1+a_{11} z^{2}+a_{12} z^{4}+\cdots\right], \\
\psi_{2}= & z^{-3 / 2}\left[1+a_{21} z^{2}+a_{22} z^{4}+\cdots\right] \\
& +b \psi_{1} \ln \left(c z^{2}\right),
\end{aligned}
$$

where the coefficients above are given by

$$
\begin{aligned}
a_{11} & =-\frac{\omega^{2}-q^{2}-2 c}{12}, \\
a_{12} & =\frac{\left(\omega^{2}-q^{2}-2 c\right)^{2}}{12 \times 32}+\frac{1}{2 z_{h}^{4}}+\frac{c^{2}}{32}, \\
a_{21} & =\frac{\omega^{2}-q^{2}-2 c}{4}, \\
b & =-\frac{\left(\omega^{2}-q^{2}\right)\left(\omega^{2}-q^{2}-4 c\right)}{32} .
\end{aligned}
$$

The coefficient $a_{22}$ is arbitrary and, in particular, we can choose $a_{22}=0$.
As it can be seen from Eq. (6), the potential $V$ vanishes when $z \rightarrow z_{h}$ so that Eq. (5) takes the form of a classical harmonic-oscillator equation with frequency $\omega$. The solutions of such equation are proportional to $\exp \left( \pm i \omega r_{*}\right)$, being denoted by $\psi_{-}$and $\psi_{+}$. The minus(plus)-sign solution can be interpreted as an ingoing (outgoing) plane wave at horizon $z=z_{h}$. The wave functions $\psi_{ \pm}$can also be expressed in terms of the normalizable and non-normalizable solutions:

$\psi_{ \pm}=\mathcal{A}_{( \pm)} \psi_{2}+\mathcal{B}_{( \pm)} \psi_{1}$

where $\mathcal{A}_{( \pm)}$and $\mathcal{B}_{( \pm)}$are connection coefficients associated to Eq. (5), and determined as functions of $\omega$ and $q$ by imposing boundary conditions on the field solution.

\section{The glueball spectral function}

\subsection{General expression}

The Minkowskian prescription of Ref. 23 can be used to compute the glueball retarded Green's function $G^{R}(\omega, q)$ and, consequently, the corresponding spectral function $\mathcal{R}=-2 \operatorname{Im} G^{R}(\omega, q)$. First we write down the on-shell version of the scalar field action (2), using the equation of motion (3):

$S_{\text {on shell }}=\left.\frac{\pi^{3} L^{5}}{4 \kappa_{10}^{2}} \int d^{4} x \sqrt{-g} e^{-\Phi} g^{z z} \phi \partial_{z} \phi\right|_{z=0}$,

So, we use the "bulk to boundary propagator" $\phi_{k}(z)$ to decompose the scalar field $\phi(z, k)$ as $\phi(z, k)=\phi_{k}(z) \phi_{0}(k)$. In terms of $\phi_{k}(z)$, the action (14) takes the form

$S_{\text {on shell }}=-\left.\int \frac{d^{4} k}{(2 \pi)^{4}} \phi_{0}(-k) \mathcal{F}(k, z) \phi_{0}(k)\right|_{z=0}$

According to the Minkowskian prescription [23, the retarded Green's function is then given by

$$
\begin{aligned}
G^{R}(k) & \equiv-\left.2 \mathcal{F}(k, z)\right|_{z=0} \\
& =\frac{\pi^{3} L^{5}}{2 \kappa_{10}^{2}} \lim _{z \rightarrow 0} \sqrt{-g} g^{z z} e^{-\Phi} \phi_{k}^{*}(z) \partial_{z} \phi_{k}(z),
\end{aligned}
$$

where the propagator $\phi_{k}(z)$ satisfies an incomingwave condition at horizon and the normalization condition $\lim _{z \rightarrow 0} \phi_{k}(z)=1$ at the boundary. 

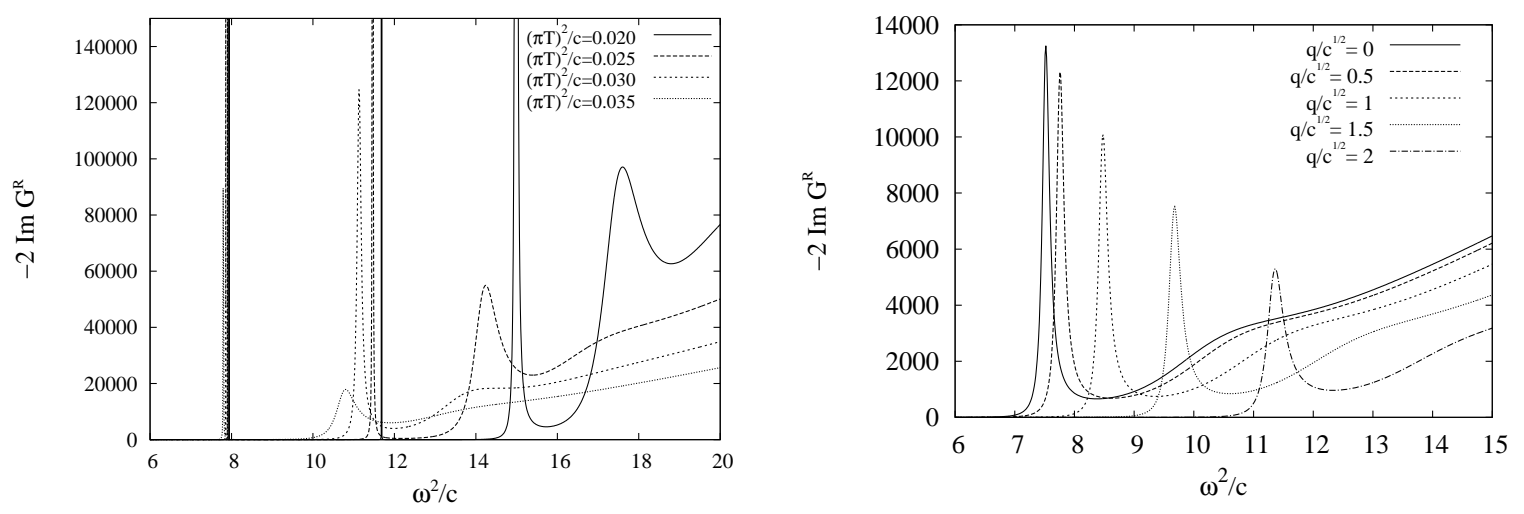

Figure 1. Left: Spectral function for $q=0$ and selected values of temperature. Right: Spectral function for $\widetilde{T}^{2}=0.05$ and selected values of the momentum. The spectral functions are in units of $N_{c}^{2} / 4 \pi^{2}$.

The function $\phi_{k}(z)$ can be expressed in terms of the wave functions defined in the last section as

$$
\begin{aligned}
\phi_{k}(z) & =z^{3 / 2} e^{\frac{c z^{2}}{2}} \frac{\psi_{-}}{\mathcal{A}_{(-)}} \\
& =z^{3 / 2} e^{\frac{c z^{2}}{2}}\left[\psi_{2}+\frac{\mathcal{B}_{(-)}}{\mathcal{A}_{(-)}} \psi_{1}\right]
\end{aligned}
$$

Then, using the relation $\pi^{3} L^{8} / 2 \kappa_{10}^{2}=N_{c}^{2} / 8 \pi^{2}$ and the expansions (7) and (8) for $\psi_{1}$ and $\psi_{2}$, we obtain

$$
\begin{aligned}
G^{R}(k)= & \frac{N_{c}^{2}}{8 \pi^{2}}\left[\left(c+2 a_{21}\right) \epsilon^{-2}+4 b \ln \left(c \epsilon^{2}\right)\right. \\
& +2\left(b+a_{21} c+a_{21}^{2}\right) \\
& \left.+4 \operatorname{Re} \frac{\mathcal{B}_{(-)}}{\mathcal{A}_{(-)}}-i \operatorname{Im} \frac{\mathcal{B}_{(-)}}{\mathcal{A}_{(-)}}\right]
\end{aligned}
$$

where $\epsilon$ is an ultraviolet regulator. In the above equation, the $\epsilon$-dependent terms are ultraviolet divergent terms that can be removed using a holographic renormalization procedure [24].

\subsection{Numerical results}

To find the glueball spectral function we need to compute the imaginary part of the retarded Green's function $G^{R}(\omega, q)$. From equation (18) it follows that

$\mathcal{R}(\omega, q)=\frac{N_{c}^{2}}{4 \pi^{2}} \operatorname{Im} \frac{\mathcal{B}_{(-)}}{\mathcal{A}_{(-)}}$.
The ratio $\mathcal{B}_{(-)} / \mathcal{A}_{(-)}$can be computed by a direct numerical integration of equation (5) following the steps described, for example, in Refs. [1825].

In figure 1, we show the dependence of the spectral function with the frequency $\widetilde{\omega}=\omega / \sqrt{c}$ for some values of temperature $\widetilde{T}=\pi T / \sqrt{c}$ and momentum $\widetilde{q}=q / \sqrt{c}$. The various peaks of $\mathcal{R}(\omega, q)$ correspond to the poles of the retarded Green's function $G^{R}$ and are interpreted as glueball states in the gauge theory. According to the gauge/gravity duality, these poles are associated with the frequencies of the black-hole quasinormal modes.

As it can be seen in the left panel of figure 1 . the number of glueball states and their lifetimes (the inverse of the half-width) decrease with the temperature. In fact, there is a temperature value $\left(\widetilde{T}^{2} \sim 0.1\right)$ from which on there are no more peaks in the spectral function, characterizing a glueball melting. For a fixed value of temperature $\widetilde{T}$, the position and the width of the peaks increase with the momentum $\widetilde{q}$, as shown in the right panel of figure 1 .

\section{From quasinormal modes to glueballs}

Two different methods have been used to compute the scalar-field QNM frequencies of the AdS black-hole spacetime (11). The first one is a series expansion method [26], which reduces the prob- 

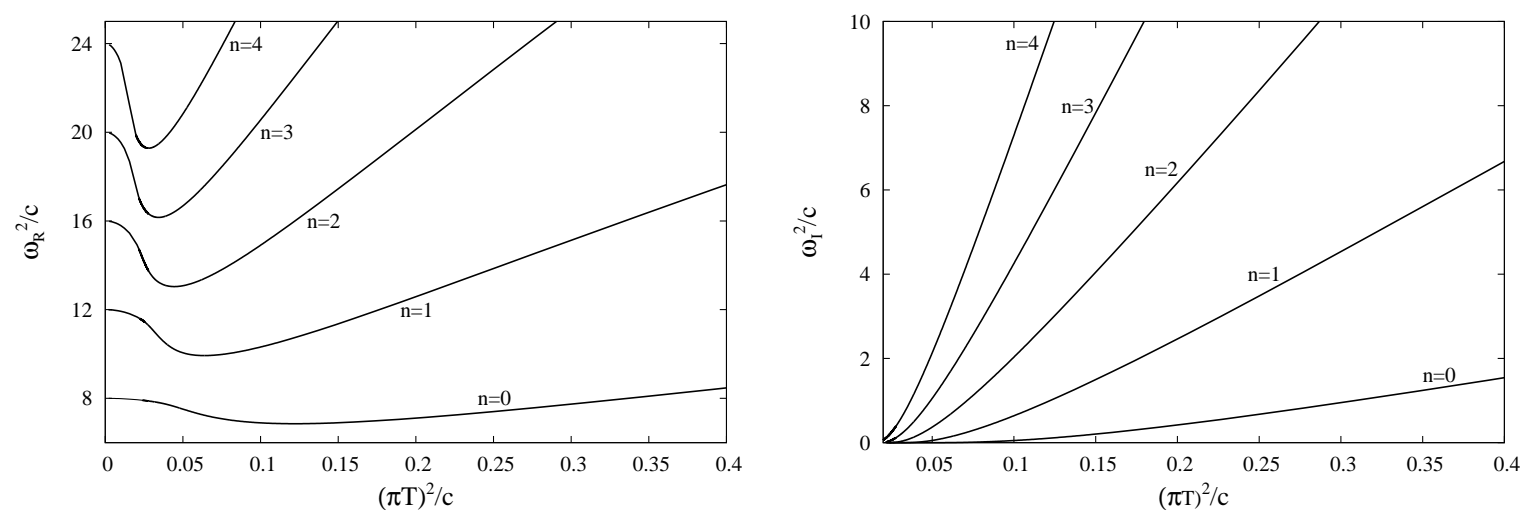

Figure 2. Numerical results for the square of the real and imaginary parts of the QN frequencies, $\omega_{R}^{2} / c$ and $\omega_{I}^{2} / c$, for the first five quasinormal modes $n=0,1, \ldots, 4$, with $q=0$.

lem to finding roots of a polynomial, and it is suitable to find QNM frequencies in the high and intermediate temperature regimes. The second method is based on the computation of BreitWigner ressonances [20, and it is efficient to investigate the spectrum for very low temperatures. The approaches are complementary and present a very good concordance in a wide range of parameter space [18.

We show in Fig. 2 the real and imaginary parts of the QNM frequencies as function of the temperature for the first five modes $n=0,1, \ldots, 4$ with $q=0$. In the limit $\widetilde{T} \rightarrow 0$, the quasinormal modes tend to the spectrum of glueballs at zero temperature: $m_{G_{n}}^{2}=4 c(n+2)$. As the temperature increases the imaginary part of the frequency $\widetilde{\omega}_{I}$ also increases, while the real part $\widetilde{\omega}_{R}$ decreases with $\widetilde{T}$. It means that the mass and the decaying time of the glueball excitations decrease with $\widetilde{T}$ for very low temperature values. In the intermediate- and high-temperature regimes, the effect of the dilaton background field is small, and the QNMs are essentially the same as those of the black hole solution with $\Phi=0$ 27/28/29]30. In particular, after reaching some minimum value the real part $\widetilde{\omega}_{R}$ increases with temperature tending asymptotically to a linear dependence. In fact, the fractional differences between the cases with and without dilaton reach a value of the order of 0.15 for $\widetilde{T}=1$, and tend asymptotically to zero in the limit of high temperatures 18 .

\section{Final comments}

In this article we review recent progress [18 on the study of the spectrum of scalar glueballs at finite temperature. The AdS/QCD soft-wall model and a black-hole background spacetime have been used to investigate thermal effects on glueball excitations of the plasma phase. As it was shown in Ref. 18, the black-hole space are associated to an unstable, metastable or stable phase of the dual theory according with the value of the temperature parameter $\widetilde{T}=T / \sqrt{c}$. It was noticed the existence of peaks in the spectral function, corresponding to glueball states, only for temperatures lower than $\widetilde{T}^{2}=0.1$, a regime for which the black hole is in an unstable phase. So according to our results, we do not expect to find glueballs in the hot plasma phase $\left(\widetilde{T}^{2}>2.39\right)$ of the QCD. Similar results were recently obtained for scalar [19] and vector 31] mesons in the soft-wall model. It would be interesting to extend these computations to other fields and other AdS/QCD models, like those of Refs. 32 33 34. Such analysis could reveal what aspects of our results are specific of the holographic model considered here and what aspects represent general characteristics of glueball excitations in the dual plasma. 


\section{Acknowledgements}

ASM and NRFB are grateful to the organizers of the "Light-Cone 2009: Relativistic Hadronic and Particle Physics" and specially to Tobias Frederico for their hospitality. The authors are partially supported by CNPq, Capes and Faperj, Brazilian agencies.

\section{REFERENCES}

1. J. M. Maldacena, Adv. Theor. Math. Phys. 2, 231 (1998) [Int. J. Theor. Phys. 38, 1113 (1999) arXiv:hep-th/9711200.

2. E. Witten, Adv. Theor. Math. Phys. 2, 253 (1998) arXiv:hep-th/9802150.

3. S. S. Gubser, I. R. Klebanov and A. M. Polyakov, Phys. Lett. B 428, 105 (1998) arXiv:hep-th/9802109.

4. H. Boschi-Filho and N. R. F. Braga, Eur. Phys. J. $\quad$ C $\quad 32, \quad 529 \quad$ (2004) arXiv:hep-th/0209080.

5. H. Boschi-Filho and N. R. F. Braga, JHEP 0305, 009 (2003) arXiv:hep-th/0212207.

6. G. F. de Teramond and S. J. Brodsky, Phys. Rev. Lett. 94, 201601 (2005) arXiv:hep-th/0501022.

7. J. Erlich, E. Katz, D. T. Son and M. A. Stephanov, Phys. Rev. Lett. 95 (2005) 261602 arXiv:hep-ph/0501128.

8. L. Da Rold and A. Pomarol, Nucl. Phys. B 721 (2005) 79 arXiv:hep-ph/0501218.

9. H. Boschi-Filho, N. R. F. Braga and H. L. Carrion, Phys. Rev. D 73, 047901 (2006) arXiv:hep-th/0507063.

10. H. Boschi-Filho, N. R. F. Braga and C. N. Ferreira, Phys. Rev. D 74, 086001 (2006) arXiv:hep-th/0607038.

11. H. Boschi-Filho, N. R. F. Braga and C. N. Ferreira, Phys. Rev. D 73, 106006 (2006) [Erratum-ibid. D 74, 089903 (2006)] arXiv:hep-th/0512295.

12. A. Karch, E. Katz, D. T. Son and M. A. Stephanov, Phys. Rev. D 74, 015005 (2006) arXiv:hep-ph/0602229.

13. P. Colangelo, F. De Fazio, F. Jugeau and S. Nicotri, Phys. Lett. B 652, 73 (2007) arXiv:hep-ph/0703316.
14. P. Colangelo, F. De Fazio, F. Giannuzzi, F. Jugeau and S. Nicotri, Phys. Rev. D 78, 055009 (2008) [arXiv:0807.1054 [hep-ph]].

15. C. P. Herzog, Phys. Rev. Lett. 98, 091601 (2007) arXiv:hep-th/0608151.

16. K. Kajantie, T. Tahkokallio and J. T. Yee, JHEP 0701, 019 (2007) arXiv:hep-ph/0609254.

17. C. A. Ballon Bayona, H. Boschi-Filho, N. R. F. Braga and L. A. Pando Zayas, Phys. Rev. D 77, 046002 (2008) arXiv:0705.1529 [hep-th]].

18. A. S. Miranda, C. A. B. Bayona, H. BoschiFilho and N. R. F. Braga, arXiv:0909.1790 [hep-th].

19. P. Colangelo, F. Giannuzzi and S. Nicotri, arXiv:0909.1534 [hep-ph].

20. E. Berti, V. Cardoso and P. Pani, Phys. Rev. D 79, 101501 (2009) arXiv:0903.5311 [grqc]].

21. H. Forkel, Phys. Rev. D 78, 025001 (2008) arXiv:0711.1179 [hep-ph]].

22. V. Mathieu, N. Kochelev and V. Vento, Int. J. Mod. Phys. E 18, 1 (2009) arXiv:0810.4453 [hep-ph]].

23. D. T. Son and A. O. Starinets, JHEP 0209, 042 (2002) arXiv:hep-th/0205051.

24. M. Bianchi, D. Z. Freedman and K. Skenderis, Nucl. Phys. B 631, 159 (2002) arXiv:hep-th/0112119.

25. D. Teaney, Phys. Rev. D 74, 045025 (2006) arXiv:hep-ph/0602044.

26. G. T. Horowitz and V. E. Hubeny, Phys. Rev. D 62, 024027 (2000) arXiv:hep-th/9909056.

27. A. O. Starinets, Phys. Rev. D 66, 124013 (2002) arXiv:hep-th/0207133.

28. A. Nunez and A. O. Starinets, Phys. Rev. D 67, 124013 (2003) arXiv:hep-th/0302026.

29. P. K. Kovtun and A. O. Starinets, Phys. Rev. D 72, 086009 (2005) arXiv:hep-th/0506184.

30. J. Morgan, V. Cardoso, A. S. Miranda, C. Molina and V. T. Zanchin, JHEP 0909, 117 (2009) arXiv:0907.5011 [hep-th]].

31. M. Fujita, K. Fukushima, T. Misumi and M. Murata, Phys. Rev. D 80, 035001 (2009) arXiv:0903.2316 [hep-ph]].

32. U. Gursoy and E. Kiritsis, JHEP 0802, 032 (2008) arXiv:0707.1324 [hep-th]]. 
33. U. Gursoy, E. Kiritsis and F. Nitti, JHEP 0802, 019 (2008) arXiv:0707.1349 [hep-th]].

34. W. de Paula, T. Frederico, H. Forkel and M. Beyer, Phys. Rev. D 79, 075019 (2009) arXiv:0806.3830 [hep-ph]]. 